\title{
Tilings of the Square with Similar Rectangles
}

\author{
M. Laczkovich ${ }^{1}$ and G. Szekeres ${ }^{2}$ \\ ${ }^{1}$ Department of Analysis, Eötvös Loránd University, \\ Múzeum krt. 6-8, 1088 Budapest, Hungary \\ laczk@ludens.elte.hu \\ ${ }^{2}$ Department of Pure Mathematics, The University of New South Wales, \\ Kensington, New South Wales 2033, Australia
}

\begin{abstract}
Let $R(u)$ denote the rectangle of sides $u$ and 1 . We prove that the square can be decomposed into finitely many rectangles similar to $R(u)$ if and only if $u$ is algebraic and each of its conjugates lies in the open half-plane $\operatorname{Re}(z)>0$.
\end{abstract}

Let $P_{1}$ and $P_{2}$ be polygons. We say that $P_{1}$ can be tiled with similar copies of $P_{2}$, if $P_{1}$ can be decomposed into finitely many nonoverlapping polygons similar to $P_{2}$. In this note we determine, for every fixed value of $u$, whether or not the square can be tiled with similar copies of the rectangle $R(u)$ of sides $u$ and 1 .

Let $T_{u}$ denote the set of positive numbers $x$ such that $R(x)$ can be tiled with similar copies of $R(u)$. Since $R(1 / x)$ is similar to $R(x)$, and $R(x+y)$ decomposes into two rectangles congruent to $R(x)$ and $R(y)$, it follows that:

(i) $u \in T_{u}$.

(ii) $x \in T_{u}$ implies $1 / x \in T_{u}$.

(iii) $x, y \in T_{u}$ implies $x+y \in T_{u}$.

Now (ii) and (iii) easily imply that if $x \in T_{u}$ and $c>0$ is rational, then $c \cdot x \in T_{u}$. Then it follows by induction on $n$ that whenever $c_{1}, \ldots, c_{n}$ are positive rational numbers, then

$$
c_{1} u+\frac{1}{c_{2} u+\frac{1}{\ddots+\frac{1}{c_{n} u}}}
$$


also belongs to $T_{u}$. Therefore, if we can find positive rational numbers such that the value of this continued fraction is 1 , then the square can be tiled with similar copies of $R(u)$. Our theorem shows that this sufficient condition is also necessary.

Theorem. For every $u>0$ the following statements are equivalent:

(i) The square can be tiled with similar copies of $R(u)$.

(ii) $u$ is algebraic and the real part of each of its conjugates is positive.

(iii) There are positive rational numbers $c_{1}, \ldots, c_{n}$ such that

$$
c_{1} u+\frac{1}{c_{2} u+\frac{1}{\ddots+\frac{1}{c_{n} u}}}=1
$$

Proof. We already proved the implication (iii) $\Rightarrow$ (i). To prove (i) $\Rightarrow$ (ii), suppose that the unit square $[0,1] \times[0,1]$ is decomposed into the nonoverlapping rectangles $R_{i}=\left[a_{i}, b_{i}\right] \times\left[c_{i}, d_{i}\right](i=1, \ldots, n)$ such that for every $i$ either $\left(d_{i}-c_{i}\right) /\left(b_{i}-a_{i}\right)$ $=u$ or $\left(d_{i}-c_{i}\right) /\left(b_{i}-a_{i}\right)=1 / u$ holds. Let $F$ and $G$ be arbitrary, complex-valued functions defined on the set $\left\{a_{i}, b_{i}, c_{i}, d_{i}: i=1, \ldots, n\right\}$. Then

$$
\Phi\left(\left[x_{1}, x_{2}\right] \times\left[y_{1}, y_{2}\right]\right) \stackrel{\text { def }}{=}\left[F\left(x_{2}\right)-F\left(x_{1}\right)\right] \times\left[G\left(y_{2}\right)-G\left(y_{1}\right)\right]
$$

defines an additive interval function and hence

$$
\sum_{i=1}^{n}\left[F\left(b_{i}\right)-F\left(a_{i}\right)\right] \times\left[G\left(d_{i}\right)-G\left(c_{i}\right)\right]=[F(1)-F(0)] \times[G(1)-G(0)]
$$

We prove that if $F$ is an arbitrary isomorphism mapping the field $\mathbf{Q}(u)$ into $\mathbf{C}$, then $\operatorname{Re} F(u)>0$. This proves (ii). Indeed, it is well known that the set of numbers $F(u)$, when $F$ runs through the isomorphisms of $Q(u)$, equals the set of all transcendental numbers if $u$ is transcendental, and equals the set of conjugates of $u$ if $u$ is algebraic. Since there are transcendental numbers with negative real part, this implies that $u$ is algebraic and each of its conjugates has positive real part; that is, (ii) holds.

Now let $F$ be an arbitrary isomorphism mapping the field $Q(u)$ into $C$, and let $v=F(u)$. We can extend $F$ to the field $K=\mathbf{Q}\left(a_{1}, b_{1}, c_{1}, d_{1}, \ldots, a_{n}, b_{n}, c_{n}, d_{n}\right)$ as an isomorphism; we also denote the extension by $F$. Let $G=\bar{F}$, then $G$ is also an isomorphism of $K$, and $G(u)=\bar{v}$. If $\left(d_{i}-c_{i}\right) /\left(b_{i}-a_{i}\right)=w_{i}$, then we have

$$
\begin{aligned}
G\left(d_{i}\right)-G\left(c_{i}\right)=G\left(d_{i}-c_{i}\right) & =G\left(\left(b_{i}-a_{i}\right) w_{i}\right)=G\left(b_{i}-a_{i}\right) \cdot G\left(w_{i}\right) \\
& =\overline{F\left(b_{i}-a_{i}\right)} \cdot G\left(w_{i}\right)=\overline{F\left(b_{i}\right)-F\left(a_{i}\right)} \cdot G\left(w_{i}\right) .
\end{aligned}
$$


Substituting these values of $G\left(d_{i}\right)-G\left(c_{i}\right)$ into (2) and taking into consideration that $F(0)=G(0)=0$ and $F(1)=G(1)=1$, we obtain

$$
\sum_{i=1}^{n}\left|F\left(b_{i}\right)-F\left(a_{i}\right)\right|^{2} G\left(w_{i}\right)=1 .
$$

For every $i$ we have either $w_{i}=u$ or $w_{i}=1 / u$. In the first case $G\left(w_{i}\right)=\bar{v}$ and $\operatorname{Re}\left(G\left(w_{i}\right)\right)=\operatorname{Re}(v)$, while in the second case $G\left(w_{i}\right)=1 / \bar{v}=v /|v|^{2}$ and $\operatorname{Re}\left(G\left(w_{i}\right)\right)=$ $\operatorname{Re}(v) /|v|^{2}$. Therefore the real part of each term on the left-hand side of (3) is of the form $c \cdot \operatorname{Re}(v)$, where $c \geq 0$. Since the value of the sum is 1 , this gives $\operatorname{Re}(v)>0$.

For the proof of (ii) $\Rightarrow$ (iii) we need the following theorem of Wall (see Theorem A of [2] or Theorem 47.1 of [3]):

Let $P(z)=z^{n}+a_{1} z^{n-1}+a_{2} z^{n-2}+\cdots+a_{n}$ be a polynomial with real coefficients, and let $Q(z)=a_{1} z^{n-1}+a_{3} z^{n-3}+a_{5} z^{n-5}+\cdots$. Then all the zeros of $P(z)$ have negative real parts if and only if

$$
\frac{Q(z)}{P(z)}=\frac{1}{c_{1} z+1+\frac{1}{c_{2} z+\frac{1}{\ddots \cdot \frac{1}{c_{n} z}}}},
$$

where the coefficients $c_{1}, c_{2}, \ldots, c_{n}$ are all positive.

Suppose that $u$ is a positive algebraic number with conjugates lying in the half-plane $\operatorname{Re}(z)>0$. Let $f$ be the minimal polynomial of $u$ and let $n$ denote the degree of $f$. We put $p(x)=(f(x)+f(-x)) / 2$ and $q(x)=(f(x)-f(-x)) / 2$; then $p$ and $q$ are polynomials with rational coefficients, $p$ is even and $q$ is odd. We have $p(u)+q(u)=f(u)=0$ and $p(u)-q(u)=f(-u) \neq 0$, as all the roots of $f$ lie in the half-plane $\operatorname{Re}(z)>0$ and $\operatorname{Re}(-u)<0$. This implies $q(u) \neq 0$ and $p(u) / q(u)=$ -1 . Now put $P(z)=p(z)-q(z)$. If $P(z)=0$, then $f(-z)=0$ and hence all the zeros of $P$ have negative real parts. Applying Wall's theorem we obtain (4), where $Q=-q$ if $n$ is even and $Q=p$ if $n$ is odd. Since the polynomials $P$ and $Q$ have rational coefficients, it follows that the numbers $c_{1}, \ldots, c_{n}$ are rational. Now, taking the reciprocal of and subtracting 1 from both sides of (4), and then substituting $z=u$, the left-hand side becomes $(P(u) / Q(u))-1$. Since $p(u)=-q(u)$, it follows that $Q(u)=-q(u)$ independently of the parity of $n$, and thus

$$
\frac{P(u)}{Q(u)}-1=-\frac{p(u)}{q(u)}=1 .
$$

Therefore (1) holds, and this completes the proof of (ii) $\Rightarrow$ (iii).

Let $T(u)$ denote the right triangle with legs $u$ and 1 . Since the diagonal divides the rectangle $R(u)$ into triangles congruent to $T(u)$, it follows from the theorem 
above that if $u>0$ is algebraic and each of its conjugates has positive real part, then the square can be tiled with similar copies of $T(u)$. In the opposite direction it was shown in Theorem 27 of [1] that if the square can be tiled with similar copies of $T(u)$, then $u$ is algebraic and each of its real conjugates is positive. We do not know whether or not this weaker condition is also sufficient for the square to be tiled with similar copies of $T(u)$. We show, however, that the cases of rectangles and triangles are indeed different; that is, there is a $u>0$ such that the square can be tiled with similar copies of $T(u)$, but cannot be tiled with similar copies of $R(u)$.

We claim that if $u$ is the (unique) real root of $f(x)=x^{3}+x-1$, then $u$ has these properties. Let the roots of $f$ be $u, v, w$. Then $u>0, w=\bar{v}$, and $u+v+$ $w=0$, therefore $\operatorname{Re}(v)=\operatorname{Re}(w)<0$. By our theorem above, this implies that the square cannot be tiled with similar copies of $R(u)$. On the other hand, a tiling with seven similar copies of $T(u)$ exists, as the following figure shows:

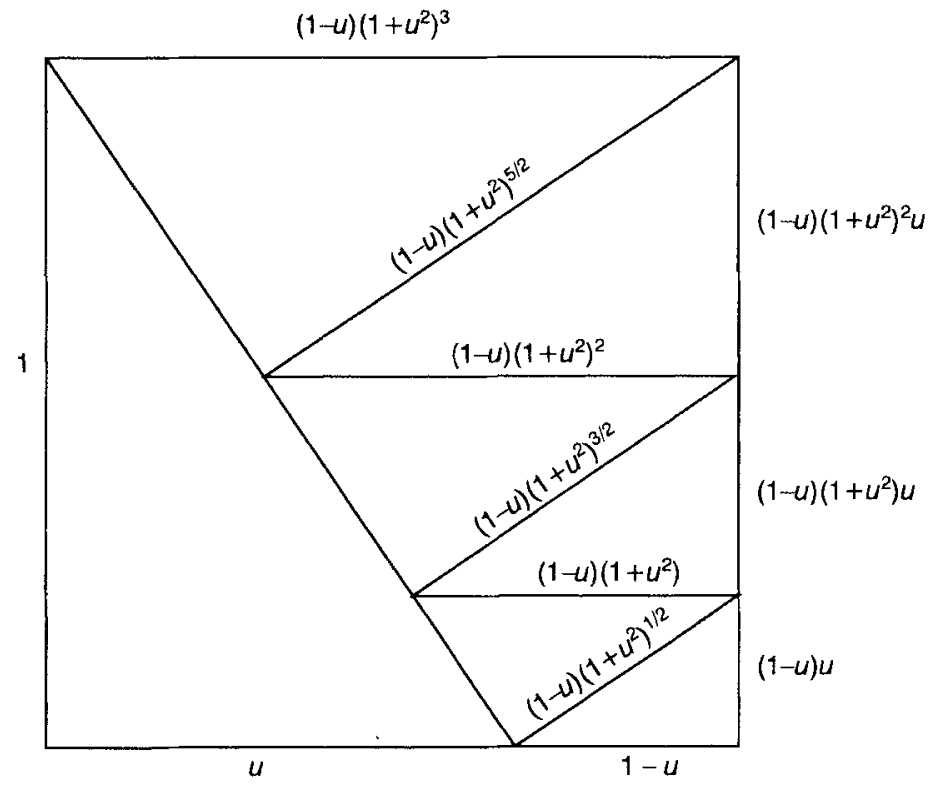

We claim that the figure is correct, that is $(1-u)\left(1+u^{2}\right)^{3}=1$. Indeed, we have $\left[1-(1-u)\left(1+u^{2}\right)^{3}\right] / u=u^{6}-u^{5}+3 u^{4}-3 u^{3}+3 u^{2}-3 u+1=\left(u^{3}+u-1\right) \times$ $\left(u^{3}-u^{2}+2 u-1\right)=0$.

\section{References}

1. M. Laczkovich, Tilings of polygons with similar triangles, Combinatorica 10 (1990), 281-306.

2. H. S. Wall, Polynomials whose zeros have negative real parts, Amer. Math. Monthly 52 (1945), 308-322.

3. H. S. Wall, Analytic Theory of Continued Fractions. Chelsea, New York, 1973.

Received January 18, 1994, and in revised form August 15, 1994. 